\title{
Detection limits of trace element analyses with the JAEA MPGA system
}

\author{
By Y. Murakamii ${ }^{1, *}$, M. Oshima², Y. Toh², A. Kimura ${ }^{2}$, M. Koizumi² ${ }^{2}$ K. Furutaka², Y. Hatsukawa², T. Kin², K. Y. Hara ${ }^{2}$, \\ K. Sushida ${ }^{3}$, M. Taniguchi ${ }^{3}$ and N. Kojima ${ }^{3}$ \\ 1 Japan Science and Technology Agency, Innovation Satellite Ibaraki, Sengen, Tsukuba, Ibaraki, 305-0047, Japan \\ 2 Japan Atomic Energy Agency, Nuclear Science and Energy Directorate, Tokai, Naka, Ibaraki 319-1195, Japan \\ 3 Toray Research Center, Inorganic Analysis Laboratories, Sonoyama, Otsu, Shiga, 520-8567, Japan
}

(Received December 3, 2009; accepted in revised form December 20, 2010)

\section{PGA / MPGA / Detection limits / Cold neutron / Coincident gamma rays}

Summary. A multiple prompt gamma-ray analysis (MPGA) system was constructed at a cold neutron beam line of JRR-3 at Japan Atomic Energy Agency (JAEA). The system consists of 8 clover Ge detectors and BGO Compton suppressors. The detection limits for 41 trace elements with this system were determined. The MPGA system has low detection limits to elements such as $\mathrm{B}, \mathrm{Sc}, \mathrm{Cd}, \mathrm{Hg}, \mathrm{Sm}$. We expect the MPGA system is a useful tool for analyses of toxic elements, heavy metals and rare-earth elements.

\section{Introduction}

The prompt gamma-ray analysis (PGA) method [1-8] has been used for the analysis of trace elements, in which prompt gamma rays emitted from excited states of nuclei after a neutron capture reaction are measured by a high-resolution gamma-ray detector, usually by a HP Ge detector. There are advantages of PGA in nondestructive and simultaneous multielemental analysis [1-8]. In order to improve the detection limit of the PGA method, we have developed a multiple prompt gamma-ray analysis (MPGA) [9-14], by applying the multiple gammaray detection technique to PGA. By detecting two or more coincident gamma rays, the signal-to-noise ratios are improved significantly. The outputs of MPGA system are composed of the energies of coincident gamma rays. They are added to a two-dimensional $\gamma-\gamma$ spectrum, which is a histogram of $\gamma-\gamma$ energy correlations. Since a nucleus produced with a neutron capture reaction often emits cascade gamma rays, peaks peculiar to nuclear species appear on the 2-D spectrum. Since the continuum background is considerably reduced as compared with 1-D spectrum, the detection limits are improved $[9,14]$. In order to study the performance of the newly constructed MPGA system, we measured detection limits for 41 elements.

\section{Experiments}

We constructed the MPGA system at a cold neutron beam line of the JRR-3 at Japan Atomic Energy Agency (JAEA). The flux of the neutron beam is about $1.4 \times 10^{7} \mathrm{n} \mathrm{cm}^{-2} \mathrm{~s}^{-1}$. The beam shape is rectangular and the size is $20 \times 30 \mathrm{~mm}$. The MPGA system consists of a beam shutter, a beam attenuator, a gamma-ray detector array, an auto-sampler, an automatic liquid nitrogen filling system [15], and a beam stopper. The details of the system have been described in Refs. $[13,14,16,17]$. The gamma-ray detector array comprises eight clover-type Ge detectors with BGO (Bismuth Germanate Oxide, $\mathrm{Bi}_{4} \mathrm{Ge}_{2} \mathrm{O}_{12}$ ) detectors. A high-speed data acquisition (DAQ) system $[18,19]$ was introduced. The arrangement of the detectors was determined through the results of GEANT4 simulations [20]. The neutron beam channel is surrounded by lithium fluoride tiles and further by lead blanks. Those shields reduce the influences of the background gamma rays and scattered neutrons. Near the measurement position, ${ }^{6} \mathrm{Li}$-enriched lithium fluoride tiles are used, which prevents the activation of the detectors by neutrons. To reduce gamma-ray background due to nitrogen nuclei in the air, the beam channel was filled with carbon dioxide gas, since carbon and oxygen nuclides have small neutron capture cross sections compared with nitrogen.

We prepared standard samples for 41 elements, i.e., B, $\mathrm{Na}, \mathrm{Si}, \mathrm{P}, \mathrm{Cl}, \mathrm{Sc}, \mathrm{Mn}, \mathrm{Fe}, \mathrm{Co}, \mathrm{Ni}, \mathrm{Zn}, \mathrm{Ge}, \mathrm{As}, \mathrm{Se}, \mathrm{Br}, \mathrm{Sr}$, Y, Mo, Ag, Cd, In, Sn, Sb, I, Ba, La, Ce, Nd, Sm, Eu, Gd, Dy, Er, Hf, Ta, Re, Os, Ir, Au, Hg, and Tl. For each element, more than two samples are prepared to derive calibration curves. The materials used were metal samples, solutions of single element samples, and oxidized or carbonated powder samples with reagent grade. (As for elements which are not available as single elements, oxidized and carbonated samples were used because oxygen and carbon nuclides have small neutron capture cross sections.) The numbers of atoms of elements in irradiation samples were estimated from those weights. The irradiation samples were wrapped with sheets of $25-\mu \mathrm{m}$-thick FEP film. Standard solution samples are dried on sheets of FEP film. The sizes of samples were smaller than $20 \times 20 \mathrm{~mm}$.

The samples are mounted in a hollow of Teflon sample holders. The size of the hollow is approximately $30 \times$

*Author for correspondence (E-mail: ym490121@u-fukui.ac.jp). 


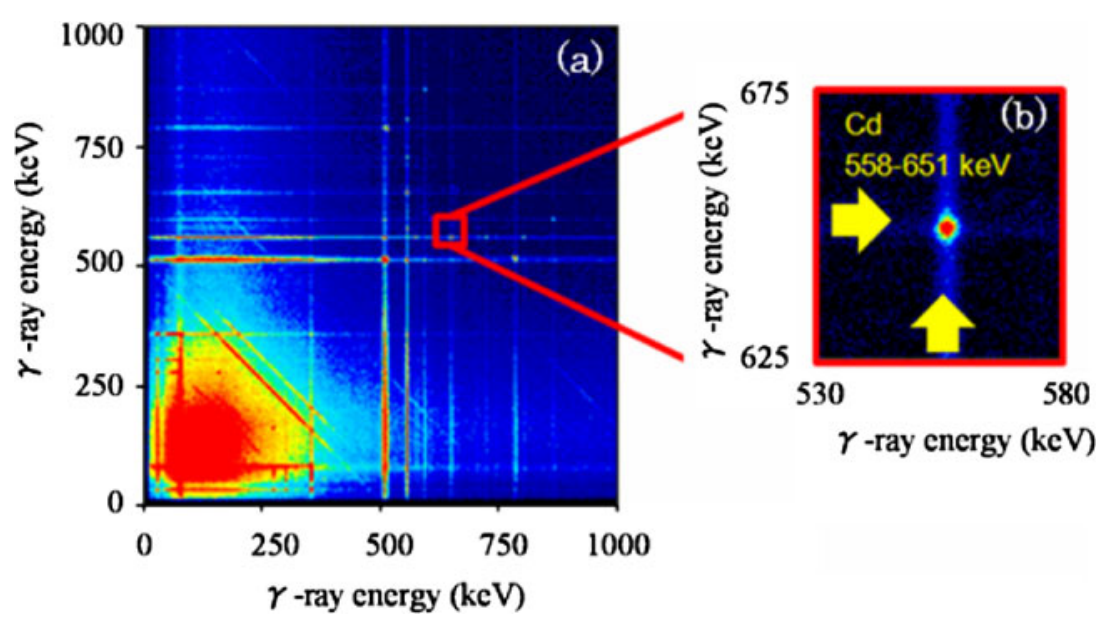

Fig. 1. (a) An example of two-dimensional $\gamma-\gamma$ spectra. An IRMM standard material sample was used as an irradiation sample. (b) An enlarged area of the figure at the cadminum peak which is the coincidence of the $558-\mathrm{keV}$ and the $651-\mathrm{keV}$ gamma rays. The gamma-ray pair is the strongest in cadmium.
$40 \mathrm{~mm}$ square. The fringes of the FEP films used to cover the samples are fixed to the holder. The samples mounted on the sample holders are sequentially transported to the measurement position by the auto-sampler, and measurements were done automatically. A weak ${ }^{133} \mathrm{Ba}$ standard radioactive source was placed on the ${ }^{6} \mathrm{LiF}$ beam duct and inside the detector array. During the measurements, the weak gamma rays were monitored simultaneously to normalize peak counts and to correct the dead time. Typical measuring time for each sample is between 600 and $10000 \mathrm{~s}$, depending on gamma-ray intensity. Before the measurements, energy calibration of Ge detectors was done with prompt gamma rays from a ${ }^{56} \mathrm{Fe}$ sample and the decay gamma rays from $\mathrm{a}^{152} \mathrm{Eu}$ standard radioactive source. Energies of gamma rays were measured below $4 \mathrm{MeV}$.

\section{Results and discussion}

Fig. 1 shows an example of two-dimensional gammagamma spectra. The vertical and horizontal axes indicate gamma energy in $\mathrm{keV}$. The intensity of gamma rays is expressed by colors. When a gamma ray was Compton scattered in a Ge detector and it escaped from the detector, the coincident events with any other gamma ray form horizontal and vertical lines. Diagonal lines are due to the Compton scattering events in which one gamma-ray is scattered in one detector and the scattered one is absorbed in another detector. Fig. $1 \mathrm{~b}$ shows a typical example of a cadmium peak.

To deduce detection limits, we measured coincident gamma rays for a known amount of single-element standard samples explained above. The counts were normalized with the counts of coincident peak of the 80- and 356-keV gamma rays from the ${ }^{133} \mathrm{Ba}$ standard source. Fig. 2 shows the calibration curve of cadmium element as an example. The cadmium calibration curve shows good linearity (coefficient of determination $R^{2}=0.999996$ ). Since the dead time itself amounts to around $30 \%$ at most, the correction utilizing ${ }^{133} \mathrm{Ba}$ has been proved to be adequate.

To check the obtained calibration curve, we measured standard samples, IRMM BCR680 and BCR681. These samples consist of various elements, the amounts of which are given by the data sheet. Table 1 shows concentration of cadmium in the samples. Our measured values with the

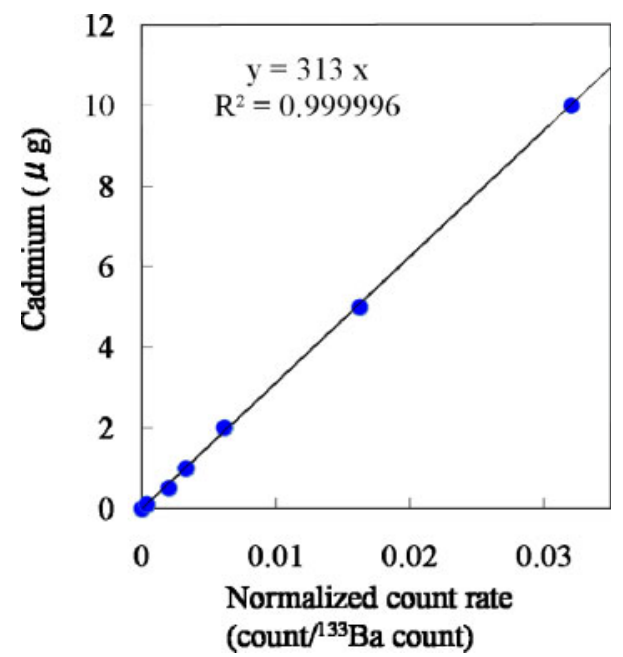

Fig. 2. The relationship between the weight of cadmium and the peak count rate. The abscissa is coincident counts of the 558- and $651-\mathrm{keV}$ gamma rays of cadmium normalized with the coincident counts of the 80- and 356-keV gamma rays of ${ }^{133} \mathrm{Ba}$.

Table 1. The concentration of cadmium in the standard material samples. The measured values with the MPGA system and cirtified values in the data sheet are given.

\begin{tabular}{lcc}
\hline Sample & This work $(\mathrm{ppm})$ & Certified value $(\mathrm{ppm})$ \\
\hline BCR680 & $139.3 \pm 2.2$ & $140.8 \pm 2.5$ \\
BCR681 & $21.5 \pm 0.8$ & $21.7 \pm 0.7$ \\
\hline
\end{tabular}

MPGA system and the certified values of the samples are tabulated. The measured values were deduced from the observed counts and the calibration curve. Those two values agree within the errors.

After the verification of the linearity of the calibration curve, we pick up data points to deduce the detection limits with the following equation:

$$
\text { Detection limit }=m \frac{3 \sigma}{C},
$$

where $m$ is the amount of the element in the sample measured, $\mathrm{C}$ is the number of $\gamma-\gamma$ coincident counts of an element, $\sigma$ is a standard deviation of the number of background 


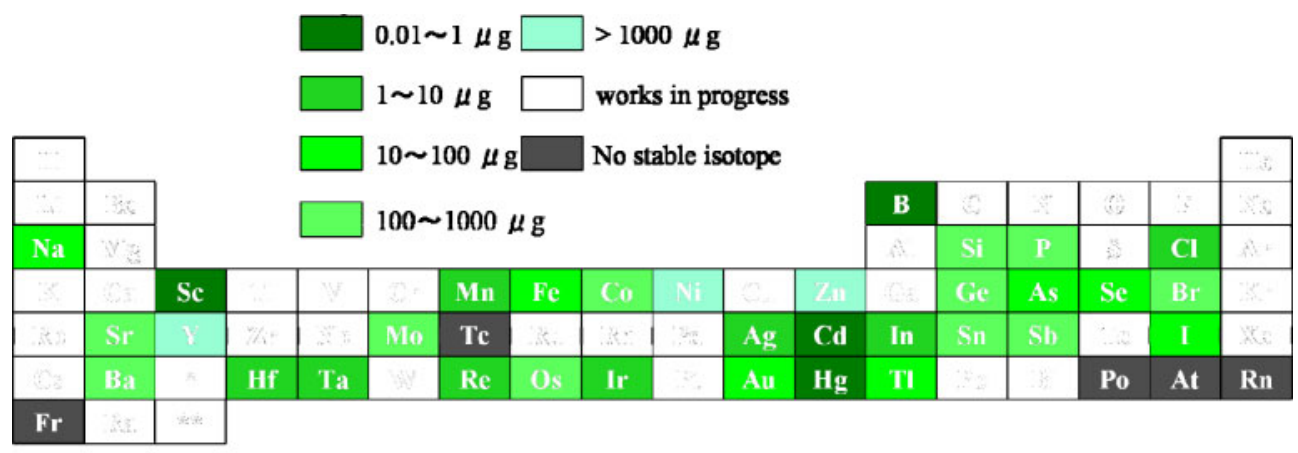

\begin{tabular}{|l|l|l|l|l|l|l|l|l|l|l|l|l|l|l|}
\hline & & & Nd & Pm & Sm & Eu & Gd & & Dy & & Er & & & \\
\hline Ac & & Pa & & Np & Pu & Am & Cm & Bk & Cf & Es & Fm & Md & No & Lr \\
\hline
\end{tabular}

Fig. 3. Detection limits for elements in standard samples with the JAEA MPGA system. counts. Using the equation, the detection limit of cadmium with our system was estimated to be approximately $0.02 \mu \mathrm{g}$.

Through the same procedure, the detection limits for the 41 single element samples in our system are obtained as shown in Fig. 3. Fig. 3 indicates that the system has low detection limits for elements such as $\mathrm{B}, \mathrm{Cl}, \mathrm{Sc}, \mathrm{Mn}, \mathrm{Ag}, \mathrm{Cd}$, In, Hf, Re, Ir, Hg, Sm, Eu, Gd, Dy and Er, which include toxic elements, heavy metals and rare-earth elements. The measurements of detection limits for the other elements are in progress.

The results of the detection limits shown in Fig. 3 are almost similar to those for PGA shown in, for example, Ref. [8]. It is rather surprising because in the case of single element samples gamma rays are dominated from those of the single element and MPGA has the demerit of count loss due to requirement of gamma-gamma coincidence. However, it is deduced that the high efficiency of our detector system compensates the count loss. As discussed in Ref. [14], an important advantage of the multiple gammaray detection method is the improvement of the $\mathrm{S} / \mathrm{N}$ ratio in a gamma-ray spectrum. This improvement is effective in determining weak peaks originating from trace elements: even when gamma-rays from major elements are strong, the resulting continuum background can be effectively reduced with this method, allowing analysis of weak gamma-rays. In other words, MPGA is expected to reduce the interference among elements contained in a sample and to bring detection limits closer to those in Fig. 3. This feature needs to be demonstrated in various combinations of major and trace elements in real samples. This work is undertaken in various fields.

\section{Summary and outlook}

We constructed a MPGA system at a neutron beam line of JRR-3 facility at JAEA. In order to study the performance of the system, we measured detection limits of the system for 41 elements. The results shows low detection limits for elements such as B, Cl, Sc, Mn, Ag, Cd, In, Hf, Re, Ir, Hg, $\mathrm{Sm}, \mathrm{Eu}, \mathrm{Gd}$, Dy and Er. This implies that MPGA method is useful for analyses of toxic elements, heavy metals and rareearth elements. Since MPGA is not sensitive to elements such as $\mathrm{C}, \mathrm{O}, \mathrm{Si}, \mathrm{Ge}, \mathrm{Co}, \mathrm{Ni}$, analyses of industrial materials are also expected, i.e., impurity analysis of semiconductors and magnetic materials, and so on.

We are planning to add four coaxial Ge detectors to the MPGA system. Since the number of coincident gamma-ray events is proportional to the square of the detection efficiency of the detector system, we expect to improve detection limits much.

Acknowledgment. This research was partly conducted as the Practical Application Research and supported by JST Innovation Satellite Ibaraki. The authors would like to thank Director Katsutoshi Goto and staff for their assistance.

\section{References}

1. Comar, D., Crouzel, C., Chasteland,M., Riviere, R., Kellershohn, C.: In: Proc. of the 1968 Intern. Conf. on Moden Trends in Activation Analysis, Vol. 1 (DeVoe, J. R., Lafleur, P. D., eds.) Nat. Bur. Stand. (US), Spec. Publ. 312 (1969), p. 114.

2. Henkelmann, R., Born, H.-J.: J. Radioanal. Chem. 16, 473 (1973).

3. Kobayashi, T., Kanda, K.: Nucl. Instrum. Methods 204, 525 (1983).

4. Lindstrom, R. M., Zeisler, R., Rossbach, M.: J. Radioanal. Chem. 112, 321 (1987).

5. Kerr, S. A., Oliver, R. A., Vittoz, P., Vivier, G., Hoyer, F., MacMahon, T. D., Ward, N. I.: J. Radioanal. Chem. 113, 249 (1987).

6. Rossbach, M.: Anal. Chem. 63, 2156 (1991).

7. Lisdstrom, R. M., Yonezawa, C.: In: Prompt Gamma Neutron Activation Analysis. (Alfassi, Z. B., Cjung, C., Eds.) CRC Press, Boca Raton, Florida (1995), p. 93.

8. Yonezawa, C., Matsue, H.: J. Radioanal. Chem. 244, 373 (2000).

9. Oshima, M., Toh, Y., Hatsukawa, Y., Hayakawa, T., Shinohara, N.: J. Nucl. Sci. Tech. 39, 292 (2002).

10. Hatsukawa, Y., Oshima, M., Hayakawa, T., Toh, Y., Shinohara, N.: Nucl. Instrum. Methods A 482, 328 (2002).

11. Oshima, M., Toh, Y., Hatsukawa, Y., Hayakawa, T., Shinohara, N.: J. Nucl. Sci. Technol. Suppl. 2, 1369 (2002).

12. Toh, Y., Oshima, M., Hatsukawa, Y., Koizumi, M., Osa, A., Kimura, A., Goto, J.: J. Nucl. Radiochem. Sci. 4, 197 (2003).

13. Toh, Y., Oshima, M., Koizumi, M. Osa, A., Kimura, A., Hatsukawa, Y.: Appl. Radiat. Isotopes 64, 751 (2006).

14. Oshima, M., Toh, Y., Hatsukawa, Y., Koizumi, M., Kimura, A., Haraga, A., Ebihara, M., Sushida, K.: J. Radioanal. Nucl. Chem. 278, 257 (2007).

15. Koizumi, M., Oshima, M., Toh, Y., Kimura, A., Kin, T., Furutaka, K., Murakami, Y., Osa, A., Ando, S.: An automatic liquidnitrogen filling system for multiple Ge detectors. Rev. Sci. Instrum. 80, 016102 (2009).

16. Oshima, M., Toh, Y., Kimura, A., Ebihara, M., Oura, Y., Itoh, Y., Sawahata, H., Matuo, M.: J. Radioanal. Nucl. Chem. 271, 317 (2007).

17. Toh, Y., Koizumi, M., Oshima, M., Kimura, A., Hatsukawa, Y., Osa, A., Goto, J.: J. Radioanal. Nucl. Chem. 272, 303 (2007). 
18. Kimura, A., Toh, Y., Koizumi, M., Furutaka, K., Kin, T., Oshima, M.: Performance of a high speed and high density data acquisition system for multiple gamma-ray detection, Conference Record of IEEE NSS/MIC/RTDS 2008, Article Number N30-68 (2008).

19. Koizumi, M., Osa, A., Toh, Y., Kimura, A., Mizumoto, M., Oshima, M., Igashira, M., Ohsaki, T., Harada, H., Furutaka, K., Saka- ne, H., Hori, J., Goto, J., Sugawara, M., Nagai, Y., Kawade, K.: Minor actinide neutron capture cross section measurements with a $4 \pi$ Ge spectrometer. Nucl. Instrum. Methods Phys. Res. A 562, 767-770 (2006).

20. Furutaka, K., Oshima, M., Kimura, A., Toh, Y., Koizumi, M., Kin, T., Goto, J.: Proc. of International Conf. on Nuclear Data for Science and Technology (2007), pp. 517-520. 